\title{
Stochastic Geometry Analysis for Mean Interference Power and Outage Probability in THz Networks
}

\author{
Joonas Kokkoniemi, Student Member, IEEE, Janne Lehtomäki, Member, IEEE, \\ and Markku Juntti, Senior Member, IEEE
}

\begin{abstract}
Mean interference power and probability of outage in the THz band (0.1-10 THz) networks are studied. The frequency band has potential for enabling future short range communication systems because of the large available spectrum resources. This can enable huge data rates, or on the other hand, large numbers of users sharing the resources. The latter case is closely related to the subject of this paper on interference modeling for dense THz networks with stochastic geometry. We use it to estimate the average behavior of random networks. The literature has shown convenient closed form solutions for the mean interference power in ultrahigh frequency band (UHF, $300 \mathrm{MHz}-3 \mathrm{GHz}$ ). Those are not always readily applicable for the THz band. This is especially the case when THz band is modeled with the molecular absorption and free space path loss. Still, the mean interference power does have closed form solutions in all cases, but in some, numerical approximations have to be used. We provide the derivation and analysis of the mean interference power and the outage probability. The results are verified with computer simulations.
\end{abstract}

Index Terms-Mean interference, outage, Poisson networks, stochastic geometry, THz communications.

\section{INTRODUCTION}

$\mathbf{S}$ PECTRUM exhaustion at the ultra high frequency band (UHF) has caused the researchers to look for opportunities at the millimeter waves (mmWaves) for the upcoming 5G networks [1]. The higher frequency bands have an advantage of the superior bandwidth, thus, data rates, when compared to the UHF band. The costs of the higher frequencies are the lower transmission radii for the base stations and users. The high propagation losses, on the other hand, enable the usage of small cells and aggressive frequency reuse. Highly directional antennas have also been seen as a way to decrease the path loss and cause less interference to the surrounding users [1].

Moving even higher in frequencies brings us to the $\mathrm{THz}$ band $(0.1-10 \mathrm{THz})$. The ever increasing demand for the higher data rates has made the $\mathrm{THz}$ band very interesting for a variety of applications, such very short range

This work was supported by the Infotech Oulu Doctoral Program and Academy of Finland under $\mathrm{SeCoFu}$ Project. The associate editor coordinating the review of this paper and approving it for publication was A. Zajic.

The authors are with the Centre for Wireless Communications, Faculty of Information Technology and Electrical Engineering, Univer-sity of Oulu, 90014 Oulu, Finland (e-mail: joonas.kokkoniemi@oulu.fi; janne.lehtomaki@oulu.fi; markku.juntti@oulu.fi). communications [2], internet of things (IoT) [3], [4], and nanocommunications [5]. In addition to the high propagation losses, there are also hardware challenges in generating high power $\mathrm{THz}$ band signals [5], [6], but progress solving those has been made [7]-[9]. Therefore, the superior bandwidth of the $\mathrm{THz}$ band transmissions will most likely be practically deployable in the coming decades. The systems can potentially reach tremendous peak data rates exceeding one terabits per second even if assuming simple pulsed communications, such as on-off keying [6]. This can be limited by the inter-symbol interference (ISI) caused by a time spread in the frequency selective channel, as in the case of the $\mathrm{THz}$ band.

Wavelengths in the $\mathrm{THz}$ band are so short that the electromagnetic energy is absorbed by the atmosphere causing huge path loss. This is caused by the $\mathrm{THz}$ band containing a large number of resonance frequencies of several molecules [6], [10], [11]. Most notably, the water vapor causes severe absorption, which, however, is very predictable based on the knowledge of the temperature and moisture of the transmission medium. Molecular absorption causes deterministic frequency selective fading to wideband signals, which is a unique feature of the $\mathrm{THz}$ band in comparison to the conventional microwave bands.

$\mathrm{THz}$ band networks have been studied in a variety of papers [12]-[15]. Because of the large path loss and absorption, the theoretical network densities can be very high [12]. We assume the number of nodes to be from less than one to about hundred per square meter. Therefore, throughout the paper, we assume the network to be interference limited. There are many papers considering the molecular noise, caused by the re-emission of the absorbed energy, e.g., [6], [16]. This could make the dense THz band networks also noise-dependent increasing the cumulated transmitted energy. However, in this paper, we assume that the noise is small in comparison to the actual transmission powers. This assumption is also supported by our previous study, which showed that the molecular noise is very weak if we consider the temporal distribution of the noise, or if we consider a case where the absorbed energy is transformed into heat [17].

A reasonable assumption of the interference limited network brings us to the main tool of this paper: the stochastic geometry, which is a powerful tool for network level problems. It can be used instead of heavy network simulations. The stochastic geometry has been studied in large numbers of papers, e.g., [18]-[29], with landmark papers by Haenggi et al. (e.g., [18]-[20]). 
The core idea of the stochastic geometry is to model the transmitter-receiver pairs in the network as a point process(es) [30]. The most common choice is to assume that the nodes are Poisson distributed because of an easy handling of the Poisson point process (PPP). It has been shown over and over again that the stochastic geometry is very useful when considering simple enough networks. On the other hand, more complex, and heterogenous networks can be modeled by superimposing multiple PPPs.

Channel power loss is usually assumed to follow simple power law, i.e., $l(r)=r^{-\alpha}$, where $l(r)$ is the path loss at distance $r$, and $\alpha$ is the path loss exponent [18], [19]. This path loss model provides closed form solutions for the aggregate interference in the network provided that the path loss exponent is large enough [18]. This is not the case in this paper, as we use the traditional free space path loss. This causes some problems with the closed form solutions, as it will be shown in Section III. Furthermore, the channel is usually assumed to be Rayleigh fading, which provides very useful simplifications, as, for instance, the outage probability can be easily calculated from the closed form solution of the aggregate interference [18].

There are a couple of works on the $\mathrm{THz}$ band mean interference and signal-to-interference-plus-noise ratio (SINR) [12]-[14]. Jornet and Akyildiz [12], Jornet [13] showed results on the stochastic interference power based on the knowledge of the number of nodes and the probability of the transmissions through the symbol length and separation. On the other hand, the work in [14] is closer to our derivation due to the utilization of the stochastic geometry. They, however, focused on the Mattern process, which assumes guard band around the transmitters, ensuring minimum distance between the nodes. This model has an advantage of not allowing the nodes to be too close to each other. This is therefore a perfect model to estimate the interference in the case where, e.g., access point locations are controlled somehow to avoid interference between them. We focus on general random networks that can be straightforwardly modeled by the Poisson process. The application of this model would be, e.g., a dense network of sensors or nanosensors randomly distributed on some small geographical area.

Because we model the $\mathrm{THz}$ band random networks with the usual $\mathrm{THz}$ channel models, i.e., the molecular absorption and general free space loss, the existing closed form solutions from the literature (e.g., [18]-[20]) are no longer applicable. Therefore, we validate all the results with the computer simulations.

Our contributions are as follows:

- We derive the mean interference power for arbitrary dimensional propagation space in the presence of molecular absorption. This can be done in two ways, either by utilizing the Campbell theorem [30], or by the derivation of the Laplace transform of the aggregated interference [18].

- Based on the mean interference level, we derive the mean signal-to-interference ratios (SIRs). We also show the outage probability of the desired link. This can be done by estimating the interference distributions. We will show

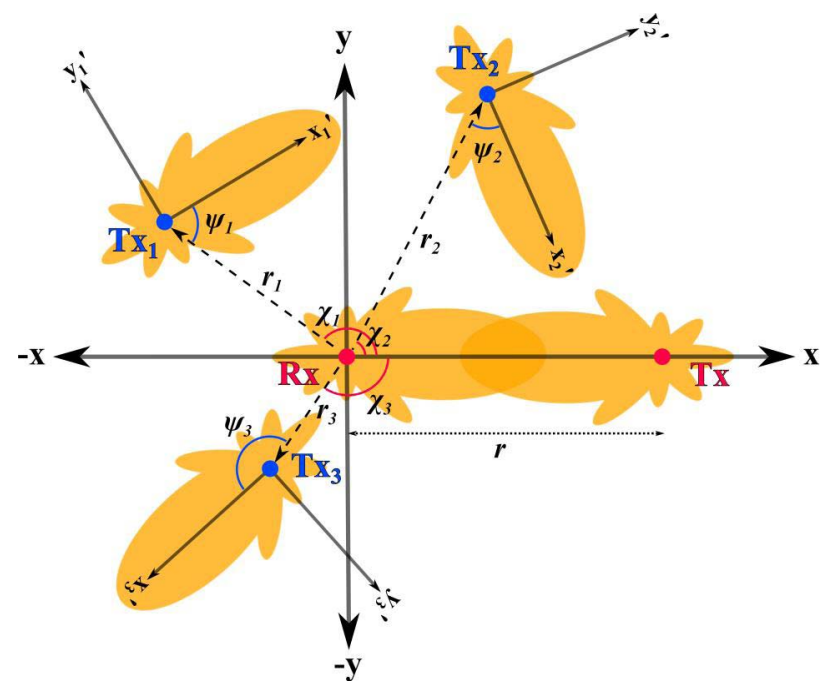

Fig. 1. System model with the receiver of interest at the origin and the corresponding transmitter on the horizontal axis.

that the interference distribution follows rather accurately the log-logistic distribution. However, better distributions can be found, but their parameter estimation is very demanding.

- We expand the discussion by utilizing directional antennas, a very likely transmission scheme for the higher frequencies.

The rest of this paper is organized as follows. The system model and its assumptions are detailed in Section II. In Section III, we derive the stochastic interference models for the $\mathrm{THz}$ band. Section IV focuses on the outage probability calculations, and directional antennas are covered in Section V. The simulation models for the validation are briefly described in Section VI. The numerical results are given in Section VII. Finally, Section VIII concludes the paper.

\section{SySTEM MODEL}

\section{A. System Geometry}

An illustration of the system model is given in Fig. 1, with three interfering transmitter-receiver node pairs as an example in addition to the receiver of interest at the origin. Because of the random network, the results hold for any point in space, but placing the studied receiver at the origin makes the notations simpler. The desired transmitter is always at a distance $r$ from the origin and located on the horizontal axis in the positive direction. The interfering nodes are randomly and evenly distributed around the origin.

We can now assume that the network follows a Poisson point process that has very important characteristics making it possible to model the interference as a homogenous PPP [19], which

1) has evenly and homogenously distributed nodes.

2) is stationary, which means that the PPP is independent of translations.

3) is simple in the sense that there cannot be multiple points at the same location.

4) is isotropic, which means that the PPP is independent on rotations. 
The above properties make the derivations feasible. For instance, we study the point at the origin without a loss of generality based on the second item. With the directional antennas, we utilize a simulation model where we rotate the coordinate axis with respect to the transmit antenna directions. The corresponding stochastic antenna gain is independent on the simulation rotations, because the PPP is independent on the rotations. The first point was already mentioned: the interfering nodes are assumed to be distributed evenly about the origin. The third fact mainly says that we can utilize $N$ random points to model an $N$ node network. The Poisson point process has further a couple of interesting features, which, however, are not utilized in this paper: an independent thinning of a PPP is PPP, and superposing multiple PPPs is PPP [19]. Technically we do use the thinning property, since we assume an ALOHA network (similarly as, e.g., in [18] and many others), i.e., all the nodes transmit randomly with a probability $p$. In other words, we basically make a $p$-fold thinning to the $N$ node network, i.e., we effectively have $p N$ nodes transmitting at the same time. Thus, the density of the network $\lambda$ is effectively $p \lambda[18]$.

\section{B. Channel Model}

Throughout the paper a line-of-sight (LOS) channels are assumed on all the links. This is a simplifying assumption which does not have an impact on the accuracy of the models since many of the non-LOS (NLOS) phenomena can be included in the absorption coefficient, or in the case of channel blocking, they can be considered as thinning processes. Thus, they do not affect the derivations of the mean interference level. However, they have an impact on the outage probability and the fading characteristics of the channel that, on the other hand, are out of scope of this paper as the aim is to focus on the impact of the molecular absorption loss on the mean interference modeling. The NLOS phenomena are briefly discussed below.

The most commonly used free space path loss model in $\mathbb{R}^{d_{s}}$ space in the $\mathrm{THz}$ band is [6], [10], [31]

$$
l(r)=\frac{\exp \left(-\kappa_{\mathrm{a}}(f) r\right)}{c_{d_{s}} d_{s} r^{d_{s}-1}},
$$

where $\kappa_{\mathrm{a}}(f)$ is the absorption coefficient, $d_{s}$ is the dimension of the space, $r$ is the distance from Tx to receiver ( $\mathrm{Rx})$, and $c_{d_{s}}$ is the volume of $d_{s}$-dimensional unit ball [31]:

$$
c_{d_{s}}= \begin{cases}\frac{\pi^{\frac{d_{s}}{2}}}{\left(d_{s} / 2\right) !} & d_{s} \text { even } \\ \frac{1}{d_{s} !} \pi^{\frac{d_{s}-1}{2}} 2^{d_{s}}\left(\frac{d_{s}-1}{2}\right) !, & d_{s} \text { odd. }\end{cases}
$$

In practical applications $c_{d_{s}}=\pi$ in $\mathbb{R}^{2}$ and $c_{d_{s}}=\frac{4}{3} \pi$ in $\mathbb{R}^{3}$. The absorption coefficient can be estimated based on the databases, such as the high-resolution transmission molecular absorption database [11]. Also, some other possible losses in the channel can be packed into the absorption coefficient, such as the scattering losses [32], [33], penetration losses [34], and surface reflection/scattering losses [35]. Then the total absorption coefficient in (1) becomes a sum over all loss coefficients

$$
\kappa(f)=\kappa_{\mathrm{a}}(f)+\kappa_{\text {others }}(f),
$$

since the overall propagation effects are multiplied in the frequency domain. This is valid for the loss mechanisms represented by the transmittance given by the Beer-Lambert law, such as the one for molecular absorption: $\exp \left(-\kappa_{\mathrm{a}}(f) r\right)$ in (1).

Several works have shown that the human body blocking (or effectively any blocking body) can cause significant losses in the high frequency channels [36]-[39]. This is a valid phenomenon also in this paper as it is not likely that all the transmitters/nodes would be in LOS condition to each other. However, the blocking can be modeled as a blocking probability in the stochastic models. Thus, as a thinning process. The blocking probability will naturally have an impact on the outage probability of the desired link. While this latter case would have an impact on the analysis here, the blocking probability does not affect on the mean interference level derivations. For this reason the body blocking is not considered in this work, but the readers can search information on it and how it is utilized in the stochastic models in the above references.

Knowing the path loss and assuming an interference limited network, we can calculate the $\operatorname{SINR} \gamma(f)$ at frequency $f$ as

$$
\gamma(f)=\frac{S(f)}{N(f)+I_{\operatorname{aggr}}(f)} \approx \frac{S(f)}{I_{\text {aggr }}(f)},
$$

where $S(f)$ is the received desired signal power, $N(f)$ is the noise power in the system, and $I_{\text {aggr }}(f)$ is the aggregated interference power at the Rx. The approximation of a small noise level is a consequence of assuming a dense network. The signal power is given by

$$
\begin{aligned}
S(f) & =P_{\mathrm{tx}}(f) G_{\mathrm{Tx}}(f) G_{\mathrm{Rx}}(f) l(r) \\
& =P_{\mathrm{tx}}(f) G_{\mathrm{Tx}}(f) G_{\mathrm{Rx}}(f) \frac{\exp \left(-\kappa_{\mathrm{a}}(f) r\right)}{c_{d_{s}} d_{s} r^{d_{s}-1}},
\end{aligned}
$$

where the transmit power density $P_{\mathrm{tx}}(f)$ is mostly normalized to unity below, and $G_{\mathrm{tx}}(f)$ and $G_{\mathrm{rx}}(f)$ are the transmitter and receiver antenna peak gains of the main lobes. We are mostly interested in the isotropic antennas, where the antenna gains are $G_{\mathrm{tx}}=G_{\mathrm{rx}}=1$, or as it will be derived below, the gains such that they integrate to unity over the propagation space. This is especially considered in the case of directional antennas in Section V. This case is also illustrated in Fig. 1, where the antenna gains are calculated from the random angles between the transmit and receive antennas.

\section{Stochastic GeOMetry for THz Networks}

Stochastic geometry is based on utilizing the stochastic average values for the interference power, which is a summation over the interfering transmitters in the network. All the calculations below are based on [18], [19], [24], [25], [30].

The aggregate interference of the network is

$$
I_{\text {aggr }}(f)=\sum_{i \in \zeta} P_{\mathrm{Tx}} l\left(r_{i}\right),
$$


where $\zeta$ is a set of interfering nodes in area/volume $|B|$ of the network. In the path loss model, we do not consider fading as a random process, since the molecular absorption causes deterministic fading given the distance $r$. Also, the $\mathrm{THz}$ transmissions are very sensitive to the existence of the LOS path and as a consequence the usual assumption of Rayleigh fading cannot be used, as discussed in the context of the outage probability analysis in Section IV.

\section{A. Modeling the Interference as a Shot Noise Process}

In order to evaluate the aggregate interference in a network, modeling the interference as a shot noise process has been widely used (e.g., [18]). The idea in the shot noise process is to model the noise components as Poisson distributed time instants. Considering a spatial random process, the time instants can be replaced with spatial locations of the nodes and the impulse responses associated with the time instants can be replaced by the path loss model. Then we can assume that (6) describes the interference as a shot noise process. This is a very handy assumption, since it allows the usage of the well known theories to analyze the aggregate interference in the network. Furthermore, if we assume Poisson distributed nodes, we can easily describe the average number of nodes in any area/volume $|B|$ with a Poisson intensity parameter $\lambda$. Assuming the ALOHA channel access for the nodes with probability $p$ to transmit, the average number of transmitting nodes is $N=p \lambda|B|$. Thus, the transmitting nodes form a Poisson distributed network with intensity $p \lambda$.

\section{B. Mean Interference Power}

The mean interference power can be evaluated in at least in two different ways: with the probability generating functionals (PGFLs), or directly with the Campbell theorem [30]. The latter is required also in the former case detailed below. Since the PGFLs are more general, we first focus on those. The PGFLs can be used to calculate the moments of a function, thus, also the mean interference power. We first calculate the Laplace transform of the aggregate interference power as given by [18] and [19]

$$
\mathcal{L}_{I_{\mathrm{aggr}}}(s)=\mathbb{E}\left[\exp \left(-s \sum_{i \in \zeta} l\left(r_{i}\right)\right)\right],
$$

where unit transmit power is assumed. Since we do not have fading as discussed above, the above equation can be written as

$$
\mathcal{L}_{I_{\text {aggr }}}(s)=\mathbb{E}_{\zeta}\left[\prod_{i \in \zeta} \exp \left(-s l\left(r_{i}\right)\right)\right] .
$$

The above expression is called the PGFL [18], which we denote as

$$
\Omega(v)=\mathbb{E}\left[\prod_{x \in \zeta} v(x)\right],
$$

where

$$
v(x)=\exp \left(-s l\left(r_{x}\right)\right)
$$

The PGFL for the PPP is further given by [18]

$$
\Omega(v)=\exp \left(-\int_{\mathbb{R}^{d_{s}}}(1-v(x)) \Lambda(x) d x\right),
$$

where $\Lambda(x)$ is the intensity function of the PPP. In the case of a homogenous Poisson process

$$
\Lambda(x)=p c_{d_{s}} d_{s} \lambda x^{d_{s}-1}
$$

with a fixed $\lambda$ over distances (homogenous network). In the sequel, we separate the propagation space and the node drop space dimensions. The propagation space has quantities $c_{d_{s}}$ and $d_{s}$ for the unit volume and the dimension of the space, respectively. For the node drop space, we use notation $c_{d_{d}}$ for the unit volume of $d_{d}$-dimensional space. This is done because a very likely scenario for the network would be a two dimensional node drop, but a three dimensional path loss. Due to usage of the unit area term $c_{d_{d}} d_{d}$, the integral over $\mathbb{R}^{d_{d}}$ is just an integration over the distance $r$. The Laplace transform of the aggregate interference becomes

$$
\begin{aligned}
& \mathcal{L}_{I_{\text {aggr }}}(s) \\
& \quad=\exp \left[-p c_{d_{d}} d_{d} \lambda \int_{\mathbb{R}^{d_{d}}}(1-\exp (-s l(r))) r^{d_{d}-1} d r\right] .
\end{aligned}
$$

The above equation has closed form solutions if the path loss exponent is larger than the dimension of the space [18]. However, because our path loss exponent follows the free space path loss, our path loss exponent is always smaller than the dimension of the space. Therefore, most of the solutions have to be numerically estimated. This is not a major problem, since it is still much faster to do the numerical computation than complete Monte Carlo system simulation. However, there is always a chance of reduced accuracy. This form though is quite accurate as it will be shown in the numerical results.

The $n$th moment of the interference power can be calculated as [21]

$$
\left.(-1)^{n} \frac{d^{n}}{d s^{n}} \mathcal{L}_{I_{\text {aggr }}}(s)\right|_{s=0} .
$$

The mean interference power becomes $-d / d s\left(\left.L_{I_{\text {aggr }}}(s)\right|_{s=0}\right)$. In the case of two dimensional node drop and three dimensional path loss, the derivative has no closed form solution, but it reduces to the Campbell theorem as seen below. In the case $d_{d}=d_{s}$, (14) does have a closed form solution:

$$
-\left.\frac{d}{d s} \mathcal{L}_{I_{\mathrm{aggr}}}(s)\right|_{s=0}=\bar{I}(f)=\frac{p \lambda}{\kappa_{\mathrm{a}}(f)},
$$

where $\bar{I}(f)$ is the mean interference power at frequency $f$ assuming unit transmit power. This special case can also be shown directly through the Campbell theorem where more general closed form solution is given. In the case of unit transmit power is not assumed, $s$ in all the above equations should be replaced with $s=P_{\mathrm{Tx}}(f) s$ [18]. Summing all the effects, the estimates for the first two moments can be easily 
estimated by calculating the derivatives of (14). Marking the Laplace transform in (13) as

$$
\mathcal{L}_{I_{\text {aggr }}}(s)=\exp (-L(s)),
$$

we can calculate rather straightforwardly the inner function derivatives:

$$
\begin{aligned}
L^{\prime}(s=0)= & P_{\mathrm{Tx}}(f) p \lambda \frac{c_{d_{d}} d_{d}}{c_{d_{s}} d_{s}} \int_{0}^{\infty} r^{d_{d}-d_{s}} \\
& \times \exp \left(-\kappa_{\mathrm{a}}(f) r\right) d r
\end{aligned}
$$

and

$$
\begin{aligned}
L^{\prime \prime}(s=0)= & P_{\mathrm{Tx}}(f) p \lambda \frac{c_{d_{d}} d_{d}}{\left(c_{d_{s}} d_{s}\right)^{2}} \int_{0}^{\infty} r^{d_{d}-2 d_{s}+1} \\
& \times \exp \left(-2 \kappa_{\mathrm{a}}(f) r\right) d r .
\end{aligned}
$$

We can calculate the mean interference power and its variance, respectively, by plugging the above expressions into the derivatives of (16)

$$
\bar{I}(f)=L^{\prime},
$$

and

$$
\operatorname{var}(I(f))=L^{\prime \prime}+\left(L^{\prime}\right)^{2} .
$$

Similar expressions can be derived for any higher moments. Also, directional antenna effects can be taken into account by multiplying the transmit power with the expected antenna gains, which is detailed in Section V.

If one is interested in the mean interference level only, the Campbell theorem offers more straightforward way to estimate it. It states that [30, p. 28]

$$
\mathbb{E}\left(\sum_{x \in \zeta} f(x)\right)=\int_{\mathbb{R}^{d_{s}}} f(x) \Lambda(x) d x .
$$

Therefore, the mean interference power becomes

$$
\bar{I}(f)=p c_{d_{d}} d_{d} \lambda \int_{\mathbb{R}^{d_{d}}} \frac{\exp \left(-\kappa_{\mathrm{a}}(f) r\right)}{c_{d_{s}} d_{s} r^{d_{s}-1}} r^{d_{d}-1} d r .
$$

We can see that when $d_{d}=d_{s}$, the equation can be solved as

$$
\bar{I}(f)=\frac{p \lambda}{\kappa_{\mathrm{a}}(f)},
$$

or

$$
\bar{I}(f)=P_{\mathrm{Tx}}(f) \frac{p \lambda}{\kappa_{\mathrm{a}}(f)}
$$

for non-unit transmit powers. The general mean interference power in the case $d_{s} \leq d_{d}$ becomes

$\bar{I}(f)=P_{\mathrm{Tx}}(f) \mathbb{E}\left[G_{\mathrm{Rx}}(f)\right] \mathbb{E}\left[G_{\mathrm{Tx}}(f)\right] p \lambda \frac{c_{d_{d}} d_{d}}{c_{d_{s}} d_{s}} \frac{\left(d_{d}-d_{s}\right) !}{\kappa_{\mathrm{a}}^{d_{d}-d_{s}+1}(f)}$,

where $\mathbb{E}\left[G_{\mathrm{Rx}}(f)\right]$ and $\mathbb{E}\left[G_{\mathrm{Tx}}(f)\right]$ are the expected antenna gains, and $\left(d_{d}-d_{s}\right)$ ! is replaced with $\Gamma\left(d_{d}-d_{s}+1\right)$, where $\Gamma(\cdot)$ is the gamma function, if non-integer path loss exponent is utilized. If $d_{s}>d_{d}$, the mean interference level does not have a closed form solution. Moreover, this latter case also requires limiting the distances of the interfering nodes around the desired receiver because of the singularity at distance zero (and close to it in the simulation models). However, in the cases where $d_{s} \leq d_{d}$ there is no such singularity as it can be seen in the above expressions.

\section{Outage Probability}

The success probability $p_{s}(f)$ of the desired transmission can be calculated with (13) by evaluating the function at point $s=\beta_{I} l(r)$, where $l(r)$ is the path loss of the desired signal and $\beta_{I}$ is the threshold for the SIR [18], i.e.,

$$
\begin{aligned}
p_{s}\left(f, \beta_{I}\right) & =\mathbb{P}\left(S(f)>\beta_{I} \bar{I}(f)\right) \\
& =\mathbb{E} \exp \left(-I_{\text {aggr }}(f) \beta l(r)^{-1}\right) .
\end{aligned}
$$

However, this is only true if the received power is exponentially distributed, i.e., Rayleigh faded. In the other words, if the signal power is exponentially distributed, the SIR distribution is exactly the Laplace transform. This is not the case in our problem. Therefore, we calculate the moments from the derivatives of the Laplace function in (15). The difficulty is to find a suitable distribution for the interference. This is not an easy task, although the moments are known. Based on our simulations, one distribution has a relatively good fit with the simulated values: the log-logistic distribution. It has parameters that can be mapped from the theoretical moments with a fairly good fit. However, it fails in the cases of very dense or thin networks. Furthermore, the log-logistic distribution gives a good fit in the case of two dimensional path loss, but fails to estimate the interference distribution for three dimensional path loss. We demonstrate this in the numerical results.

The log-logistic distribution is characterized by the probability density function [40]

$$
f(x)=\frac{(\beta / \alpha)(x / \alpha)^{\beta-1}}{\left(1+(x / \alpha)^{\beta}\right)^{2}},
$$

where $\alpha$ is the scale parameter, and $\beta$ is the shape parameter. These can be solved numerically from the moments of the interference distribution and the moments of the log-logistic distribution [40]

$$
\mathbb{E}\left[X^{k}\right]=\alpha^{k} \frac{k \pi / \beta}{\sin (k \pi / \beta)} .
$$

Given the the interference distribution, the outage probability is

$$
p_{o}(S(f), \beta)=1-p_{s}\left(f, \beta_{I}\right)=1-\int_{0}^{S(f) / \beta_{I}} f_{I}(x) d x,
$$

where $f_{I}(x)$ is the probability density function of the interference power of the desired node.

\section{Directional Antennas}

Directional antennas are often envisioned being utilized in the higher frequencies because of the large path loss, but also because of the potential benefit of large multiple input multiple 
output (MIMO) transmission gains [41]. MIMO configurations would also be a natural way to realize directional antennas.

The above stochastic geometry analysis with isotropic antennas means that the path loss has unity term for the antenna gains $G_{\mathrm{Rx}}(f, \Psi, \Phi)$ and $G_{\mathrm{Tx}}(f, \Psi, \Phi)$ for the $\mathrm{Rx}$ and Tx, respectively, in all the directions $(\Psi, \Phi)$, where $\Psi$ is the azimuth angle and $\Phi$ is the elevation angle. There have been some works on directional antennas for the stochastic geometry, e.g., [28], [29].

When we take into account the directional antennas, we have to consider two cases: 1) the total transmit power is the same as in the isotropic case, and 2) it is different to the total isotropic power. Both cases are rather straightforward extensions. The expected effect of the directional antennas can be modeled by the expected antenna gain. Assuming the same pattern for the Tx and Rx ends, and a unit integral over the total transmit power over the antenna radiation pattern, the antenna gain averaged over all angles becomes

$$
\begin{aligned}
\mathbb{E}_{\Theta} & {\left[G_{\mathrm{Rx}}(f, \Theta)\right] } \\
= & \mathbb{E}_{\Theta}\left[G_{\mathrm{Tx}}(f, \Theta)\right]=\frac{G_{\mathrm{i}}}{c_{d_{s}} d_{s}} \int_{\mathbb{R}^{d_{s}}} A(f, \Theta) d \Theta,
\end{aligned}
$$

where $A(f, \Theta)$ is the antenna pattern, $G_{\mathrm{i}}$ is the gain compared to the total isotropic power, and $\Theta$ is the azimuth/elevation angle dependent on the dimensions of the system. The term $G_{i}$ is included just to isolate the two cases above, i.e., $G_{\mathrm{i}}=1$ for case one, and something else for the second case. If we assume isotropic antennas, the above equation yields an expected gain of $1 / c_{d_{s}} d_{s}$ in all directions, since the total transmit power is obtained from

$$
P_{\mathrm{Tx}} \int_{\mathbb{R}^{d_{s}}} \frac{1}{c_{d_{s}} d_{s}} d \mathbb{R}^{d_{s}}=P_{\mathrm{Tx}}
$$

as $P_{\mathrm{Tx}}$ sets the limit for the total transmit power (assuming there is no additional gain).

Case one above yields the same values for the expected antenna gains as in the isotropic case, because the total transmit power and average radiated/receiver power are the same due to random angles to and from the interfering transmitters. Then results in Section VII demonstrate that the expected interference is indeed the same if the transmit powers are the same. Regardless of the same interference, the antenna gain provides $\max _{\theta}\left(G_{\mathrm{Tx}}(f, \Theta)\right) \times \max _{\theta}\left(G_{\mathrm{Rx}}(f, \Theta)\right)$ times better SIR, because the desired link power is higher (recall Fig. 1 and the assumption on the desired link antenna directions).

If the additional expected antenna gain is less than, or larger than the effective isotropic gain, it naturally has an impact on the received average interference power. However, the SIR remains the same if the antenna pattern remains the same because the desired link power decreases or increases with the gain. In any case, the theoretical effect of the expected antenna gains can be included in the mean interference similarly as in
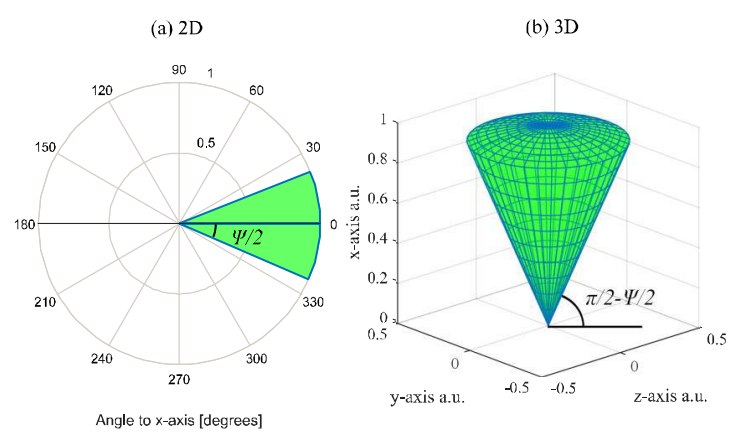

Fig. 2. Antenna patterns with (a) 2D, and (b) 3D path loss.

the case of free space path loss. The mean gain becomes

$$
\begin{array}{r}
\bar{I}(f)={ } c_{d_{d}} d_{d} \lambda \mathbb{E}\left[G_{\mathrm{Rx}}(f)\right] \mathbb{E}\left[G_{\mathrm{Tx}}(f)\right] \\
\times \int_{\mathbb{R}^{d_{d}}} \frac{\exp \left(-\kappa_{\mathrm{a}}(f) r\right)}{c_{d_{s}} d_{s} r^{d_{s}-1}} r^{d_{d}-1} d r .
\end{array}
$$

The validity of this approach will be shown in the section on the numerical results, where we assume symmetrical antenna response in the elevation and azimuth directions. The utilized antenna patterns in two and three dimensional spaces are shown in Fig. 2. The antenna gain is always assumed to point towards the positive horizontal axis. Thus, the coordinate axes are rotated according to the system geometry. The figure does not take into account the actual gains in the direction of the antenna patterns. These are not the most realistic antenna patterns, but they allow easy validation due to their straightforward mathematical representation. If the antenna half beam width is $\Psi / 2$, then the gains toward these angles become

$$
G_{2 \mathrm{D}}(f,-\Psi / 2: \Psi / 2)=\frac{1}{\Psi},
$$

for the two dimensional (2D) path loss, and

$$
G_{3 \mathrm{D}}(f,-\Psi / 2: \Psi / 2)=\frac{1}{2 \pi(1-\cos (\Psi / 2))},
$$

for the three dimensional (3D) path loss. Please notice that the $3 \mathrm{D}$ antenna gain is defined only about the azimuth angle $\Psi$. The property will be explained in more detail the next section, but it is related to the fact that the simple antenna patterns can be represented in 2D space and by scaling as above and by scaling with the probability of the antenna pointing in certain direction (as done below). The latter term comes from the expression for the area of a spherical cap, which also defines the steradian of the angle around the cone set by the antenna half beam. Both gains reduce to isotropic gains if the antenna half beam is set to $\Psi / 2=\pi$.

The expected antenna gains for the cases of interest become

$$
G(f)=G_{2 \mathrm{D}}(f,-\Psi / 2: \Psi / 2)\left(\frac{\Psi}{2 \pi}\right)^{2},
$$

for a 2D node drop and 2D path loss assuming identical gain pattern in the both $\mathrm{Rx}$ and Tx. The term $\Psi / 2 \pi$ is the 
probability that the $\mathrm{Tx} / \mathrm{Rx}$ points at the $\mathrm{Rx} / \mathrm{Tx}$ in a $2 \mathrm{D}$ path loss model. Similarly, the expected antenna gains for 2D drop and 3D path loss, and 3D node drop and 3D path loss become, respectively,

$$
\begin{aligned}
& G(f)=G_{3 \mathrm{D}}(f,-\Psi / 2: \Psi / 2)\left(\frac{\Psi}{2 \pi}\right)^{2}, \\
& G(f)=G_{3 \mathrm{D}}(f,-\Psi / 2: \Psi / 2)\left(\frac{1}{2}(1-\cos (\Psi / 2))\right)^{2},
\end{aligned}
$$

where $0.5(1-\cos (\Psi / 2))$ is the probability that the $\mathrm{Tx} / \mathrm{Rx}$ points at the $\mathrm{Rx} / \mathrm{Tx}$ in the $3 \mathrm{D}$ path loss model. The 2D node drop with 3D path loss is similar to the 2D/2D case because we assume the interfering links, as well as the desired link, are parallel to the node drop plane. If the desired link, or the interfering links, would point at random direction(s), the expected interference would be $G_{3 \mathrm{D}}(f,-\Psi / 2$ : $\Psi / 2)\left(\frac{\Psi}{2 \pi}\right) \frac{1}{2}(1-\cos (\Psi / 2))$. However, it is unlikely the links would point in random elevation directions if all the nodes are on a plane. The fourth case of a 3D node drop and 2D path loss model is not very likely in any realistic systems, but the expected antenna gain can be calculated similarly as in the $2 \mathrm{D} / 2 \mathrm{D}$ case, but with three dimensional antenna gain (or the same as 2D/3D).

\section{Simulation Model}

As mentioned in the previous section, the simulation model is based on two dimensional antenna patterns. This can be done due to utilization of a dot product to calculate the angle between the positive $\mathrm{x}$-axis and the random point in space and because of the assumed perfectly symmetrical antenna patterns.

Regardless of the dimension of the space, the interfering nodes are distributed evenly around the origin (i.e., the desired receiver) from 0 to $400 \mathrm{~cm}$ distances. Thus, the $400 \mathrm{~cm}$ radius defines the node drop area or volume as a circle or a ball. With the parameters used in the numerical results, even smaller radius, such as $100 \mathrm{~cm}$ radius (with reasonable transmit powers), would ensure small interference contribution from outside the simulation area. However, $400 \mathrm{~cm}$ radius makes sure that no interference arrives from outside this area. This can be seen in the numerical results, where simulation results on the mean interference match perfectly with those given by the theories (infinite area).

The nodes are distributed in spherical coordinates based on the disc/sphere point picking principle to achieve an even distribution for the nodes. In the case of isotropic antennas, it is enough to calculate the Euclidian distances from random points to the origin due to equal antenna gain in all directions. The distances and the antenna gains can then be input into the channel model

$$
l(r)=\frac{\exp \left(-\kappa_{\mathrm{a}}(f) r\right)}{c_{d_{s}} d_{s} r^{d_{s}-1}} G_{\mathrm{Rx}}(f) G_{\mathrm{Tx}}(f)
$$

and the total interference is given by (6) with the average number of $N$ interfering nodes drawn from the Poisson distribution taking into account the transmit probability $p$, effectively thinning the Poisson process.
In the case of directional antennas, the antenna directions (azimuth/elevation) are randomly chosen based on the disc/sphere point picking principle again to ensure even distribution for the directions. Next we can calculate the angles from the origin to the random points, as well as the angles from the random points with random coordinate axes to the origin. We have to calculate the position of the origin from the new random coordinate axes of the interfering nodes. This is a straightforward negation of the coordinates of the random nodes, i.e., if every interfering node is considered to lie in its own origin, then the original origin is at $\left(x^{\prime}, y^{\prime}, z^{\prime}\right)=$ $(-x,-y,-z)$ from the nodes. This, however, means all the coordinate axes are oriented similarly. To take into account the rotations by the random transmit angles, we apply the rotation operation(s) to the coordinates to calculate the real location of the original origin in the new coordinate axes. The rotations can be done in many ways, but we utilize rotation matrices to calculate the coordinates

$$
\left[\begin{array}{l}
x^{\prime} \\
y^{\prime}
\end{array}\right]=\left[\begin{array}{cc}
\cos (2 \pi-\theta) & -\sin (2 \pi-\theta) \\
\sin (2 \pi-\theta) & \cos (2 \pi-\theta)
\end{array}\right]\left[\begin{array}{l}
-x \\
-y
\end{array}\right]
$$

for the $2 \mathrm{D}$ node drop and

$$
\begin{aligned}
{\left[\begin{array}{l}
x^{\prime} \\
y^{\prime} \\
z^{\prime}
\end{array}\right]=} & {\left[\begin{array}{ccc}
\cos (2 \pi-\phi) & 0 & \sin (2 \pi-\phi) \\
0 & 1 & 0 \\
-\sin (2 \pi-\phi) & 0 & \cos (2 \pi-\phi)
\end{array}\right] } \\
& \times\left[\begin{array}{ccc}
\cos (2 \pi-\theta) & -\sin (2 \pi-\theta) & 0 \\
\sin (2 \pi-\theta) & \cos (2 \pi-\theta) & 0 \\
0 & 0 & 1
\end{array}\right]\left[\begin{array}{l}
-x \\
-y \\
-z
\end{array}\right]
\end{aligned}
$$

for the 3D node drop, where the right-hand matrix rotates the coordinate axes about the $\mathrm{z}$-axis, and the left-hand matrix rotates the coordinate axes about the y-axis. The subtraction of the random angles from $2 \pi$ shifts the rotation matrix from rotating the point in the coordinate axis in the counterclockwise direction to rotating the coordinate axis in the counterclockwise direction. This is important with respect to the point mapping, since the trigonometric functions rotate in a counterclockwise direction. Thus, it is more convenient that the coordinate axes rotate in this direction in this intermediate phase. Based on the new coordinates, we can calculate the angles from the origin to the nodes with a dot product

$$
\vartheta_{\mathrm{Rx}}=\cos ^{-1}\left(\frac{x}{\sqrt{x^{2}+y^{2}+z^{2}}}\right),
$$

where $\cos ^{-1}(\cdot)$ is the inverse cosine function. Similarly, the angles from the nodes to the origin can be calculated

$$
\vartheta_{\mathrm{Tx}}=\cos ^{-1}\left(\frac{x^{\prime}}{\sqrt{x^{\prime 2}+y^{\prime 2}+z^{\prime 2}}}\right),
$$

assuming the transmitters and the receiver at the origin are pointed at the positive $\mathrm{x}$-axis (a unit vector along the positive $\mathrm{x}$-axis). Then the angles for the antenna patterns and the Euclidian distances can be input into the path loss model. Two dimensional antenna patterns can be used even in three dimensional space. The reason for this is the increased probability for the higher angles due to additional degree of freedom 
TABLE I

PARAMETERS USED IN THE NUMERICAL ResUlTS

\begin{tabular}{|c|c|c|}
\hline \hline Quantity & Symbol & Value \\
\hline \hline Absorption coefficient & $\kappa_{\mathrm{a}}$ & $0.1 \mathrm{~cm}^{-1}$ (if fixed) \\
\hline Antenna beam width & $\Psi$ & $\pi / 4$ \\
\hline $\begin{array}{c}\text { Node densities in 2D space } \\
\text { (50 to 5000 nodes) }\end{array}$ & $\lambda$ & 1.0 to 99.5 per $\mathrm{m}^{2}$ \\
\hline $\begin{array}{c}\text { Node densities in 3D space } \\
\text { (50 to 5000 nodes) }\end{array}$ & $\lambda$ & 0.2 to $18.7{\text { per } \mathrm{m}^{3}}^{2}$ \\
\hline Probability to transmit & $p$ & 1 \\
\hline Transmit power & $P_{\mathrm{Tx}}(f)$ & $1 \mathrm{~W} / \mathrm{Hz}$ \\
\hline Transmitter-receiver distance & $r$ & $10 \mathrm{~cm}$ \\
\hline \hline
\end{tabular}

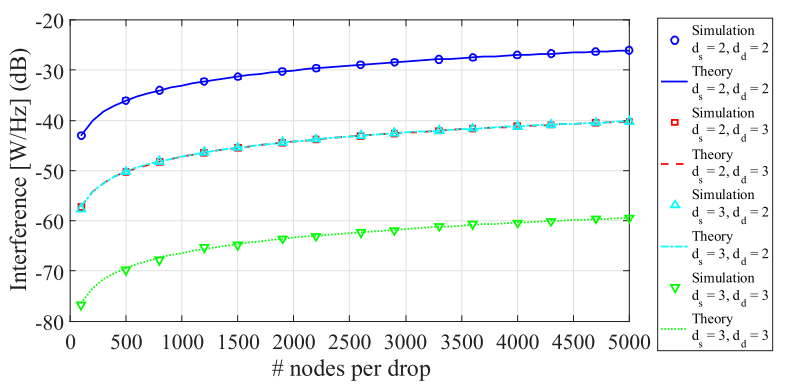

Fig. 3. Mean interference power versus the number of nodes in the network.

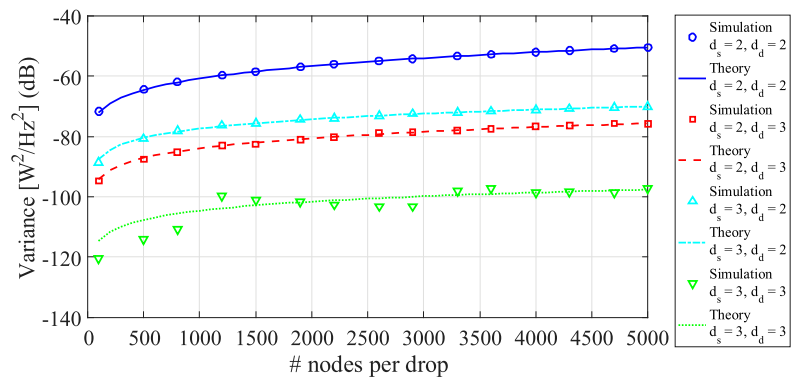

Fig. 4. The variance of the mean interference power versus the number of nodes in the network.

provided by the elevation angle. Of course, this procedure depends on the antenna patterns, and this assumption can only be used with symmetric antenna patters as in our case.

\section{NUMERICAL RESULTS}

Numerical results for the mean interference power (also with directional antennas) and outage probabilities are given in this section. The utilized parameters are given in Table I. The given absorption coefficient is utilized for the most of the results except for the frequency domain results where realistic absorption coefficients are used. The results are given without the antenna aperture term. Therefore, results represent the interference intensity at location of the desired receiver.

\section{A. Mean Interference Power}

The mean interference power and the corresponding variance are calculated with (19) and (20) and compared against the raw moments of the simulation data given by $\mathbb{E}\left[I_{\text {sim }}^{k}\right]$ with the results shown in Figs. 3 and 4, respectively. The simulation data was obtained with the simulation model presented in the

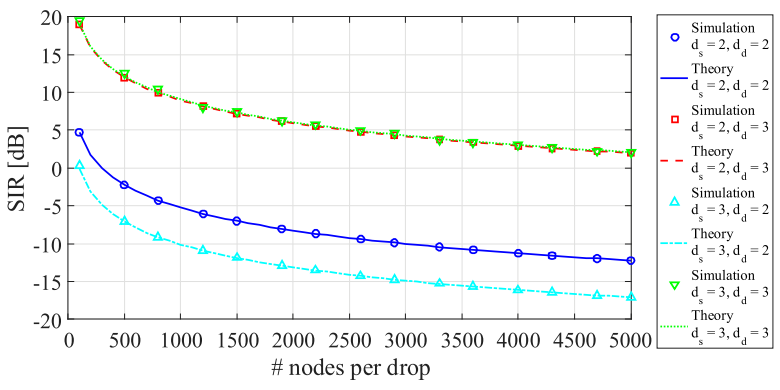

Fig. 5. SIR versus the number of nodes in the network.

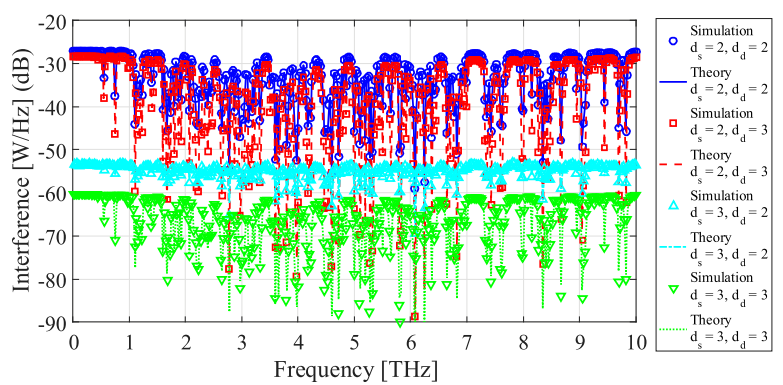

Fig. 6. Mean interference power as a function of frequency for 100 nodes in the network.

previous section. Similarly to the directional antenna cases, the isotropic antenna gain is assumed to sum unity over all directions. Figs. 3 and 4 show a very good agreement between the theory and the simulation for both the mean and the variance. The full 3D case suffers from a larger variation in variance caused by the relatively thinner network and in general lower interference power because of the larger path loss. Still, the results give a perfect match, also in the case of SIR in Fig. 5. The SIR was calculated with the path loss model given in (1) and with the parameters given in Table I. The relatively short desired link distance combined with large losses from the distant interferers leads to reasonable SIR values for lower number of users (still hundreds), and even to good SIR levels in the three dimensional node drop cases. Still, a very large number of interferers causes the SIR to drop considerably. In the next section we will see that if the absorption is increased, the SIR will increase in some frequencies because of the reduced interference. Secondly, we assume that all the nodes are transmitting all the time, which represents the worst case scenario. In reality, the SIR would be better as the simple sensor nodes would most likely be idle most of the time. The overall picture shows that the medium can be shared with very large number of users because of the large propagation loss in the channel.

\section{B. Frequency Domain Mean Interference}

The distinguishing feature of the $\mathrm{THz}$ band to the lower frequency bands is the strong molecular absorption loss. Therefore, instead of using just the fixed absorption coefficient, Figs. 6 and 7 give the mean interference power and SIR for 100 nodes in the network as a function of frequency. The absorption coefficients were calculated by using the HITRAN catalogue [11] and the well known theories for the transmittance [6], [10]. 


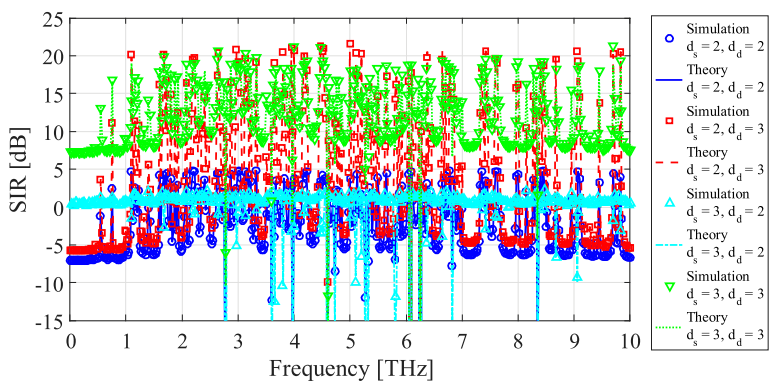

Fig. 7. SIR as a function of frequency for 100 nodes in the network.

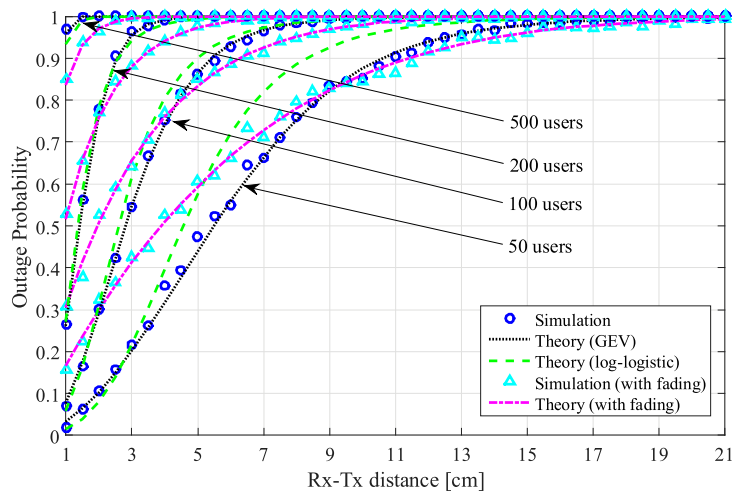

Fig. 8. Outage probabilities and the simulation data for 2D node drop and 2D path loss versus the desired link's Rx-Tx distance.

Figs. 6 and 7 show what can be expected: where the absorption is strong, the interference power drops as the atmosphere absorbs the energy. This may or may not lead to better SIR levels depending on the absorption experienced by the desired link. As it can be seen in Fig. 7, deep fades in general cause better SIR, theoretically allowing better quality pointto-point links with fixed node density, or denser networks in the case of fixed target SIR level. However, deep fades in the desired link also have an impact on the link quality and can take the SIR down even if the interference level would be low.

\section{Outage Probability}

The outage probabilities were calculated with (29) for the non-fading case and with (26) for the fading case. As it was mentioned earlier, the log-logistic distribution is not a perfect solution for describing the general interference distribution. A perfect distribution would be a three parameter generalized extreme value (GEV) distribution [42], but it requires simulation data for parameter fitting.

Results in Figs. 8-11 comprise the simulation results of the probability of outage for the non-fading and the fading cases (unit mean Rayleigh fading), as well as the theoretical results given by the log-logistic distribution, Eq. (26), and the GEV (with simulation aided parameters). Interestingly, as predicted by the previous works, the Laplace transform of the aggregate interference power gives the outage distribution in the presence of fading also if there is molecular absorption loss.

As it was discussed above, the GEV gives a perfect distribution for the interference distribution without fading. A fairly good option is given by the log-logistic distribution,

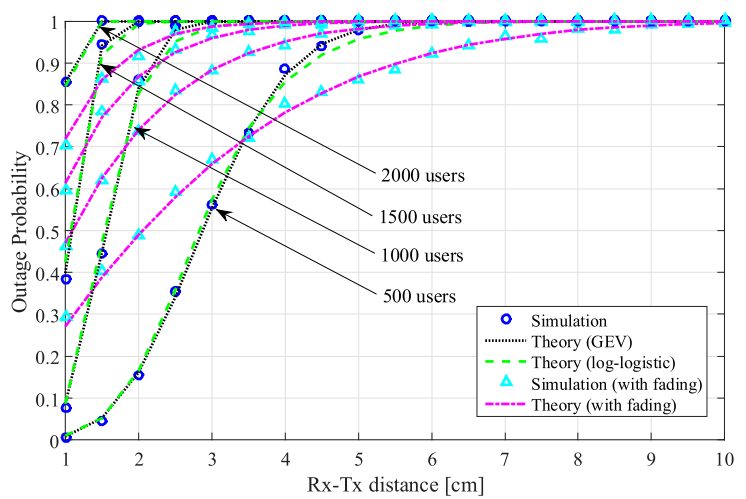

Fig. 9. Outage probabilities and the simulation data for 3D node drop and 2D path loss versus the desired link's Rx-Tx distance.

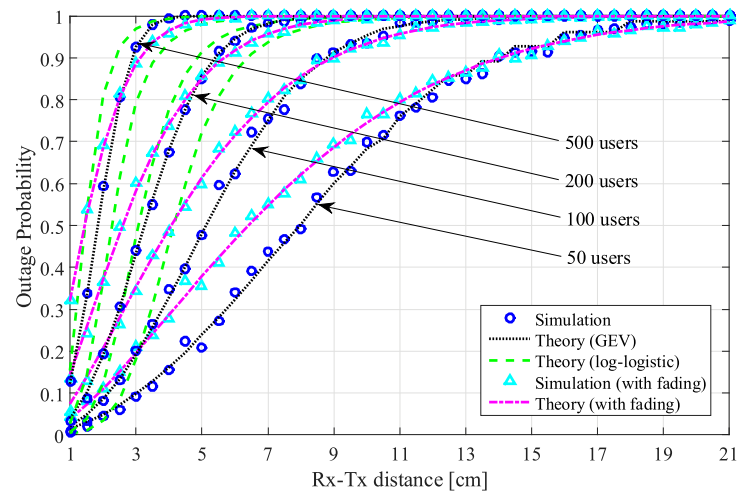

Fig. 10. Outage probabilities and the simulation data for 2D node drop and 3D path loss versus the desired link's Rx-Tx distance.

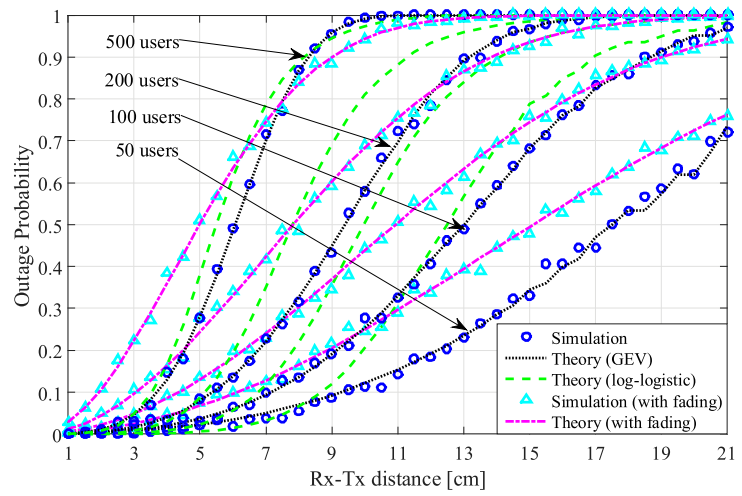

Fig. 11. Outage probabilities and the simulation data for 3D node drop and 3D path loss versus the desired link's Rx-Tx distance.

which can be mapped directly from the raw moments from the theory. It gives a good fit in the case of $2 \mathrm{D}$ path loss (see Figs. 8 and 9), with limitations in the accuracy in the case of very thin networks. When using the 3D path loss model, it mostly fails to predict the interference distribution, particularly if the density is too low or too high. Therefore, the interference distributions in the THz band are subject of further investigation in the future. This is a very important issue, since the proper distributions combined with the theories would give all the required information on the collective network behavior.

\section{Directional Antennas}

Mean interference powers and SIRs for the directional antennas are given in Figs. $12-15$ based on the models in 


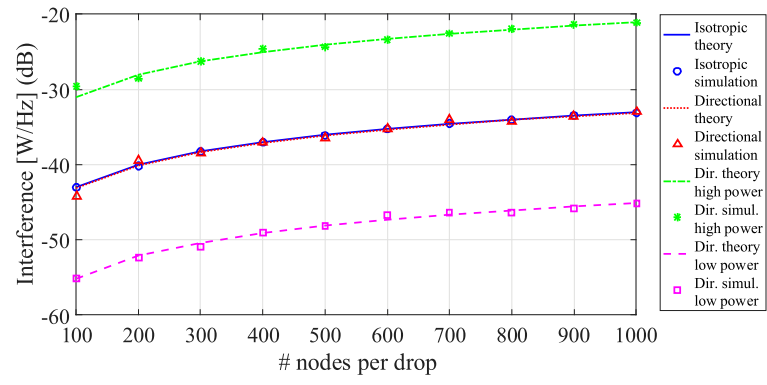

Fig. 12. Mean interference power for 2D node drop and 2D path loss versus the number of nodes in the network for various antenna configurations.

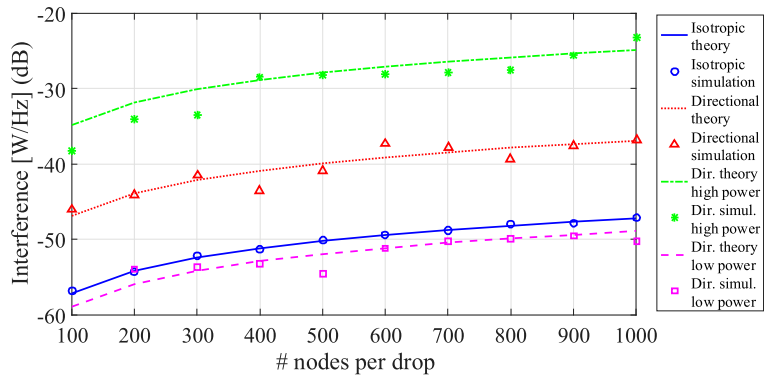

Fig. 13. Mean interference power for 2D node drop and 3D path loss versus the number of nodes in the network for various antenna configurations.

Section V. There are two observations to be made from the results. Firstly, if the effective isotropic radiated power is the same, the directional antennas do not decrease or increase the interference power. This is because the expected antenna gains are equivalent in both the isotropic and the directional antennas. An exception to this is made by the 2D node drop and 3D path loss. In this case, and with our assumptions, the antenna patterns are parallel to the plane. Therefore, the probability of receiving interference from the plane is the same as with the two dimensional path loss, however, with three dimensional path gain on the antenna. This is clearly visible in Fig. 13, which shows approximately $10 \mathrm{~dB}$ larger interference power for the directional antenna case in comparison to the isotropic case. This is a direct consequence of the probabilities to receive interference, which are (similarly as in (36) and (37)) $\Psi / 2 \pi$ for the $2 \mathrm{D}$ case and $0.5(1-\cos (\Psi / 2))$ for the 3D case. In this case the expected increase of the interference when moving to the directional antennas is $(\Psi / 2 \pi)^{2} /[0.5(1-\cos (\Psi / 2))]^{2}=10.79 \equiv$ $10.33 d B$ with $\Psi=\pi / 4$.

In the general case, if the total power is increased or decreased with respect to the isotropic power, it naturally has an impact on the total interference power, as it can be seen in Figs. 12-13 (four-fold increase and decrease in power for high and low power cases, respectively). This leads us to the second interesting finding: effective isotropic power has no impact on the SIR, because we effectively scale both the desired and the interfering links. This follows naturally from the assumption of an interference limited network. Although the directional antennas have no impact on the total interference power in the network (given equal total transmit power in the isotropic case), they do give a large gain in the SIR performance because of the increased desired link power, which is assumed to be perfectly aligned in these examples. As discussed earlier, this

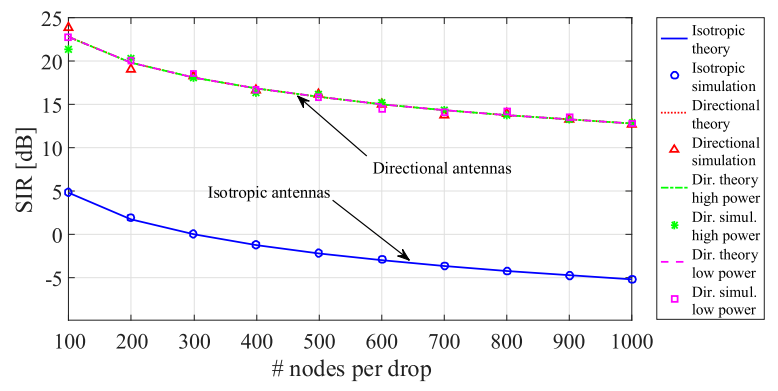

Fig. 14. SIR for $2 \mathrm{D}$ node drop and $2 \mathrm{D}$ path loss versus the number of nodes in the network for various antenna configurations.

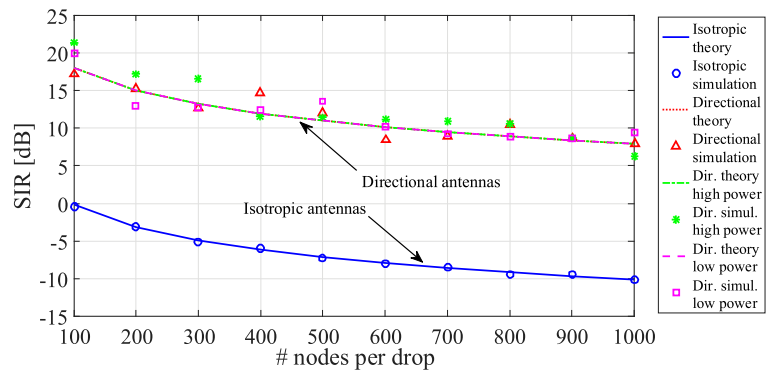

Fig. 15. SIR for $2 \mathrm{D}$ node drop and $3 \mathrm{D}$ path loss versus the number of nodes in the network for various antenna configurations.

is a very likely scenario in the $\mathrm{THz}$ band because of the large path loss. Directional links will make it significantly easier to mitigate the large losses in the channel, as it can also be seen here. Of course, they reflect the effects of very simple system geometry with a simple antenna pattern. Regardless of this, the results here show that stochastic geometry can be very efficiently used in the $\mathrm{THz}$ band network interference modeling.

\section{DISCUSSION AND CONCLUSION}

We have shown that the stochastic geometry can be utilized in the network analysis for dense $\mathrm{THz}$ networks, such as nanonetworks. The moments of the interference power can be reliably estimated based on the stochastic models presented herein. Similarly, the outage probabilities can be estimated based on the analysis. Still, the full evaluation of the outage performance will require considerable future work on the interference distributions. For instance, the GEV distribution gives a perfect fit to the simulations, but requires simulation data for the parameter fitting. Instead, the interference distribution should be parameterized from the theoretical moments, or directly from the Laplace transform.

Directional antennas were also included in the analysis. This is very important in the case of higher frequencies, as the high loss links will almost surely require highly directional antennas to offer reliable communications. We utilized simple and illustrative antenna patterns, leaving room for future work on more realistic ones. However, the antenna pattern has no impact on the mean interference level if the effective isotropic power remains constant. Thus, more complex antenna patterns can be utilized in the place of very simple ones. The directional antennas and possible side lobes do have an impact on the interference distributions because the variance of the interference increases. This requires further work in the future. 
Another point for future work is the fading in the THz channels. The molecular absorption causes deterministic frequency selective fading, which also causes delayed signal components. The possible NLOS phenomena will further increase the delayed signal components. Unlike in the lower frequencies, these components are not summed with the LOS response, but with later pulses because of the very short pulses lengths in the $\mathrm{THz}$ band (picoseconds). Therefore, the existing models for the fading, such as Rayleigh and Ricean models, are not applicable. These require a lot of work in the future if NLOS paths are modeled properly.

The overall analysis herein shows that the stochastic geometry can very efficiently be used even at very high frequencies. Many of the envisioned applications for the $\mathrm{THz}$ frequencies include dense sensor networks. The stochastic geometry will therefore offer a very good set of tools to model the interference in those.

\section{ACKNOWLEDGMENT}

J. Kokkoniemi would like to thank the following foundations for their support: Riitta ja Jorma J. Takasen säätiö, Walter Ahlströmin säätiö, Tauno Tönningin säätiö, Ulla Tuomisen säätiö, and Nokia Foundation.

\section{REFERENCES}

[1] T. S. Rappaport et al., "Millimeter wave mobile communications for IEEE access cellular: It will work!" IEEE Access, vol. 1, no. 1, pp. 335-349, May 2013.

[2] I. F. Akyildiz, J. M. Jornet, and C. Han, "Terahertz band: Next frontier for wireless communications," Elsevier Phys. Commun., vol. 12, pp. 16-32, Sep. 2014.

[3] I. F. Akyildiz and J. M. Jornet, "The Internet of nano-things," IEEE Wireless Commun. Mag., vol. 17, no. 6, pp. 3-19, Dec. 2010.

[4] J. M. Jornet and I. F. Akyildiz, "The Internet of multimedia nano-things in the terahertz band," in Proc. Eur. Wireless Conf., 2012, pp. 1-8.

[5] I. F. Akyildiz and J. M. Jornet, "Electromagnetic wireless nanosensor networks," Nano Commun. Netw., vol. 1, no. 1, pp. 3-19, Mar. 2010.

[6] J. M. Jornet and I. F. Akyildiz, "Channel modeling and capacity analysis for electromagnetic nanonetworks in the terahertz band," IEEE Trans. Wireless Commun., vol. 10, no. 10, pp. 3211-3221, Oct. 2011.

[7] I. Llatser, C. Kremers, A. Cabellos-Aparicio, J. M. Jornet, E. Alarćon, and D. N. Chigrin, "Graphene-based nano-patch antenna for terahertz radiation," Photon. Nanostruct. Fundam. Appl., vol. 10, no. 4, pp. 353-358, Oct. 2012.

[8] P. K. Singh, G. Aizin, N. Thawdar, M. Medley, and J. M. Jornet, "Graphene-based plasmonic phase modulator for terahertz-band communication," in Proc. Eur. Conf. Antennas Propag., 2016, pp. 1-5.

[9] J. M. Jornet and I. F. Akyildiz, "Graphene-based plasmonic nanotransceiver for terahertz band communication," in Proc. Eur. Conf. Antennas Propag., 2014, pp. 492-496.

[10] S. Paine, "The am atmospheric model," Smithsonian Astrophys. Observ., Cambridge, MA, USA, Tech. Rep. 152, 2012.

[11] L. S. Rothman et al., "The HITRAN 2008 molecular spectroscopic database," J. Quant. Spectrosc. Radiat. Transf., vol. 110, nos. 9-10, pp. 533-572, Jun./Jul. 2009.

[12] J. M. Jornet and I. F. Akyildiz, "Information capacity of pulse-based wireless nanosensor networks," in Proc. IEEE Int. Conf. Sens., Commun. Netw., Jun. 2011, pp. 80-88.

[13] J. M. Jornet, "Fundamentals of electromagnetic nanonetworks in the terahertz band," Ph.D. dissertation, Dept. Elect. Eng., Georgia Inst. Technol., Atlanta, GA, USA, Dec. 2013.

[14] V. Petrov, D. Moltchanov, and Y. Koucheryavy, "Interference and SINR in dense terahertz networks," in Proc. IEEE Veh. Technol. Conf. (VTC Fall), Sep. 2015, pp. 1-5.

[15] V. Petrov, D. Moltchanov, and Y. Koucheryavy, "On the efficiency of spatial channel reuse in ultra-dense THz networks," in Proc. IEEE Global Commun. Conf., Dec. 2015, pp. 1-7.

[16] P. Wang, J. M. Jornet, M. A. Malik, N. Akkari, and I. F. Akyildiz, "Energy and spectrum-aware MAC protocol for perpetual wireless nanosensor networks in the terahertz band," Ad Hoc Netw., vol. 11, no. 8, pp. 2541-2555, Nov. 2013.
[17] J. Kokkoniemi, J. Lehtomäki, and M. Juntti, "A discussion on molecular absorption noise in the terahertz band," Elsevier Nano Commun. Netw. J., vol. 8, pp. 35-45, Jun. 2016.

[18] M. Haenggi and R. K. Ganti, "Interference in large wireless networks," Found. Trends Netw., vol. 3, no. 2, pp. 127-248, Nov. 2008.

[19] M. Haenggi, J. G. Andrews, F. Baccelli, O. Dousse, and M. Franceschetti, "Stochastic geometry and random graphs for the analysis and design of wireless networks," IEEE J. Sel. Areas Commun., vol. 27 , no. 7, pp. 1029-1046, Sep. 2009.

[20] M. Haenggi, "Outage, local throughput, and capacity of random wireless networks," IEEE Trans. Wireless Commun., vol. 8, no. 8, pp. 4350-4359, Aug. 2009.

[21] H. Elsawy, E. Hossain, and M. Haenggi, "Stochastic geometry for modeling, analysis, and design of multi-tier and cognitive cellular wireless networks: A survey," IEEE Commun. Surveys Tuts., vol. 15, no. 3, pp. 996-1019, 3rd Quart., 2013.

[22] M. Haenggi, Stochastic Geometry for Wireless Networks. Cambridge, U.K.: Cambridge Univ. Press, 2013.

[23] P. Nardelli, "Analysis of the spatial throughput in interference networks," Ph.D. dissertation, Dept. Commun. Eng., Univ. Oulu, Oulu, Finland, 2013

[24] J. Venkataraman, M. Haenggi, and O. Collins, "Shot noise models for outage and throughput analyses in wireless ad hoc networks," in Proc. IEEE Military Commun. Conf., Oct. 2006, pp. 1-7.

[25] F. Baccelli and B. Blaszczyszyn, "Stochastic geometry and wireless networks, volume I-Theory," Found. Trends Netw., vol. 3, nos. 3-4, pp. 249-449, Dec. 2009.

[26] P. Cardieri, "Modeling interference in wireless ad hoc networks," IEEE Commun. Surveys Tuts., vol. 12, no. 4, pp. 551-572, 4th Quart., 2010.

[27] M. Z. Win, P. C. Pinto, and L. A. Shepp, "A mathematical theory of network interference and its applications," Proc. IEEE, vol. 97, no. 2, pp. 205-230, Feb. 2009.

[28] J. Wildman, P. Nardelli, M. Latva-Aho, and S. Weber, "On the joint impact of beamwidth and orientation error on throughput in directional wireless poisson networks," IEEE Trans. Wireless Commun., vol. 13, no. 12 , pp. 7072-7085, Dec. 2014

[29] O. Georgiou, S. Wang, M. Z. Bocus, C. P. Dettmann, and J. P. Coon, "Directional antennas improve the link-connectivity of interference limited ad hoc networks," in Proc. Int. Symp. Pers. Indoor Mobilem Radio Commun., 2015, pp. 1311-1316.

[30] A. Baddeley, "Stochastic geometry," in Spatial Point Processes and Their Applications (Lecture Notes in Mathematics), W. Weil, ed. Berlin, Germany: Springer, 2007.

[31] S. Weber and J. G. Andrews, "Transmission capacity of wireless networks," Found. Trends Netw., vol. 5, nos. 2-3, pp. 109-281, Jan. 2012.

[32] J. Kokkoniemi, J. Lehtomäki, K. Umebayashi, and M. Juntti, "Frequency and time domain channel models for nanonetworks in terahertz band," IEEE Trans. Antennas Propag., vol. 63, no. 2, pp. 678-691, Feb. 2015.

[33] M. Franceschetti, "Stochastic ray pulse propagation," IEEE Trans. Antennas Propag., vol. 52, no. 10, pp. 2742-2752, Oct. 2004.

[34] J. Kokkoniemi, J. Lehtomäki, and M. Juntti, "Measurements on penetration loss in terahertz band," in Proc. Eur. Conf. Antennas Propag., 2016, pp. 1-5

[35] J. Kokkoniemi, J. Lehtomäki, and M. Juntti, "Measurements on rough surface scattering in terahertz band," in Proc. Eur. Conf. Antennas Propag., Apr. 2016, pp. 1-5.

[36] K. Venugopal, M. C. Valenti, and R. W. Heath, Jr., "Interference in finitesized highly dense millimeter wave networks," in Proc. Inf. Theory Appl. Workshop, Feb. 2015, pp. 175-180.

[37] K. Venugopal and R. W. Heath, Jr., "Millimeter wave networked wearables in dense indoor environments," IEEE Access, vol. 4, pp. 1205-1221, Mar. 2016.

[38] "Location based performance model for indoor mmWave wearable communication," in Proc. IEEE Int. Conf. Commun., May 2016, pp. 1-6.

[39] M. Gapeyenko, A. Samuylov, M. Gerasimenko, D. Moltchanov, and S. Singh, "Analysis of human-body blockage in urban millimeter-wave cellular communications," in Proc. IEEE Int. Conf. Commun., May 2016, pp. 1-7.

[40] F. Ashkar and S. Mahdi, "Fitting the log-logistic distribution by generalized moments," Elsevier J. Hydrol., vol. 328, nos. 3-4, pp. 694-703, Sep. 2006.

[41] I. F. Akyildiz and J. M. Jornet, "Realizing ultra-massive MIMO $(1024 \times 1024)$ communication in the $(0.06-10)$ terahertz band," Elsevier Nano Commun. Netw. J., vol. 8, pp. 46-54, Jun. 2016.

[42] T. G. Bali, "The generalized extreme value distribution," Elsevier Econ. Lett., vol. 79, no. 3, pp. 423-427, Jun. 2003. 